\title{
Sound radiation due to modal vibrations of a spherical source in an acoustic quarterspace
}

\author{
Seyyed M. Hasheminejad* and Mahdi Azarpeyvand \\ Department of Mechanical Engineering, Iran University of Science and Technology, Narmak, Tehran 16844, Iran
}

Received 7 September 2002

Revised 22 February 2004

\begin{abstract}
Radiation of sound from a spherical source, vibrating with an arbitrary, axisymmetric, time-harmonic surface velocity, while positioned within an acoustic quarterspace is analyzed in an exact manner. The formulation utilizes the appropriate wave field expansions along with the translational addition theorem for spherical wave functions in combination with the classical method of images to develop a closed-form solution in form of infinite series. The analytical results are illustrated with numerical examples in which the spherical source, vibrating in the pulsating $(n=0)$ and translational oscillating $(n=1)$ modes, is positioned near the rigid boundary of a water-filled quarterspace. Subsequently, the basic acoustic field quantities such as the modal acoustic radiation impedance load and the radiation intensity distribution are evaluated for representative values of the parameters characterizing the system.
\end{abstract}

\section{Introduction}

There exists a vast amount of literature on scattering theory, extending back for more than a century and across the boundaries of many disciplines such as acoustics, electromagnetics, and quantum mechanics. In particular, acoustic scattering (radiation) by two or more closely positioned interacting spherical bodies has been the subject of several works during the last 40 years [1-3]. A classic acoustic radiation problem is that of computing the coupling between two, identical, constant volume velocity, very small, time harmonic spherical radiators. Karnovskii [4,5] expressed this coupling as a modified complex radiation load on one of the sources. Ingard and Lamb [6] computed the modified output from one of the sources which is also a measure of the modified resistive radiation loading. They actually treated the equivalent problem of a single source in the vicinity of an infinite, perfectly reflecting, rigid boundary. They then employed image theory to replace th $t$ of the boundary by an image source, thereby converting their problem to that considered by Karnovskii.

In references [4-6], the authors assumed that the sources are so small relative to the wavelength that one does not significantly perturb the sound field radiated by the other. Hence, it is natural to ask how the radiation loading changes if the spherical sources are sufficiently large so that one does perturb the field radiated by the other. An approach to solving this problem is to utilize the translational addition theorem for spherical wave functions [7] to replace wave functions that represent the radiation from one sphere by an equivalent set of wave functions centered at the other one. Using this method, axisymmetric acoustic radiation from a spherical source vibrating with an arbitrary, time-harmonic velocity distribution while positioned wholly outside a fluid sphere is examined in [8]. Likewise, axisymmetric acoustic coupling between two finite-sized spherical sources is studied in [9,10]. Reference [11] investigates acoustic radiation from a spherical sour dded eccentrically within a fluid sphere and vibrating with an arbitrary, axisymmetric, time-harmonic velocity distribution. In a more recent paper [12], this analysis is generalized for description of non-axisymmetric acoustic field problems involving non-concentric spherical boundaries. In a

*Corresponding author. Tel.: +9821 73912957; +9821 2557166; Fax: +9821 7451143; E-mail: hashemi@iust.ac.ir. 


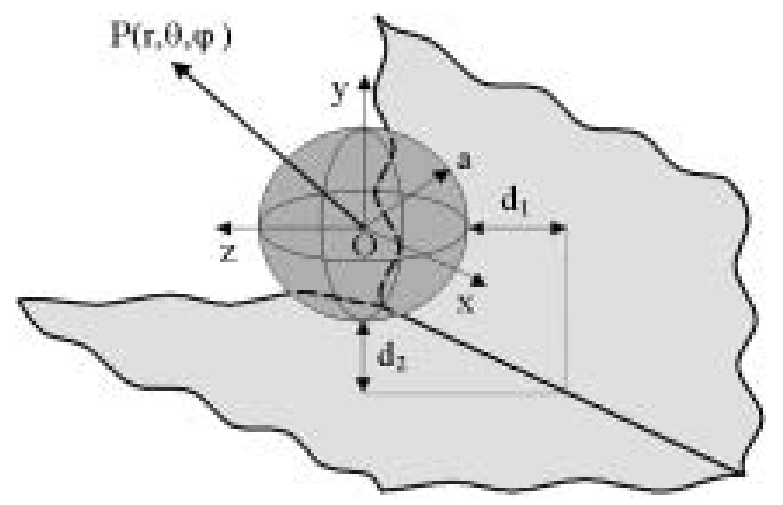

Fig. 1. Problem geometry.

companion paper [13], numerical results are presented for the specific case of two identical monopole sources within a fluid sphere that is embedded in another infinite fluid medium.

In this work, the analysis in [9] is extended for a situation involving multiple spherical radiators where the simplifying assumption of axisymmetry about a common axis does not exist. In particular, the method of images [14, 15] is used to study harmonic acoustic radiation from a modally vibrating spherical source that is located near a rigid corner Fig. 1. The presented analysis can lead to a better understanding of dynamic response of near-corner radiators that are of practical interest in room acoustics, outdoor acoustics, and noise control engineering.

\section{Formulation}

The problem can be analyzed by means of the standard methods of theoretical acoustics. The fluid is assumed to be inviscid and ideal compressible that cannot support shear stresses making the state of stress in the fluid purely hydrostatic. In view of the fact that the spherical source is supposed to undergo time-harmonic surface oscillations, with frequency $\omega$, the field equations may conveniently be expressed in terms of a scalar velocity potential as [15]:

$$
w=-\nabla \Phi, p=-i \omega \rho \Phi, \nabla^{2} \Phi+k^{2} \Phi=0
$$

where $i=\sqrt{-1}, \rho$ is the ambient fluid density, $w$ is the fluid-particle-velocity vector, $p$ is the acoustic pressure in the inviscid fluid, $k=\omega / c$ is the acoustic wave number, $c$ is the ideal speed of sound, and we have assumed harmonic time variations throughout with $e^{-i \omega t}$ dependence suppressed for simplicity.

The geometry and the coordinate systems of the problem are depicted in Fig. 2. The origin $O_{1}$ of the spherical coordinate system $\left(r_{1}, \theta_{1}, \varphi_{1}\right)$ is placed at the center of the real sphere. The origins $O_{\alpha}(\alpha=2,3,4)$ of the spherical coordinate systems $\left(r_{\alpha}, \theta_{\alpha}, \varphi_{\alpha}\right)$ are placed at the centers of the image spheres. These centers are separated so that $l_{12}=l_{21}=l_{34}=l_{43}=2\left(a+d_{1}\right)$ and $l_{13}=l_{31}=l_{24}=l_{42}=2\left(a+d_{2}\right)$. The dynamics of the multi-scattering problem may be expressed in terms of four scalar potentials corresponding to the waves radiating from each sphere, i.e.,

$$
\Phi_{(\alpha)}\left(r_{\alpha}, \theta_{\alpha}, \varphi_{\alpha}, \omega\right)=\sum_{n=0}^{\infty} \sum_{m=-n}^{n} x_{n m}^{(\alpha)}(\omega) h_{n}\left(k r_{\alpha}\right) P_{n}^{m}\left(\cos \theta_{\alpha}\right) \mathrm{e}^{i m \varphi_{\alpha}} \quad(\alpha=1,2,3,4)
$$

where $h_{n}(x)=j_{n}(x)+i y_{n}(x)$ is spherical Hankel function of order $n$ [16], $P_{n}^{m}(x)=(-1)^{m}(1-$ $\left.x^{2}\right)^{m / 2}\left[d^{m} P_{n}(x) / d x^{m}\right]$ is the associated Legendre function, and $x_{n m}^{(1)}(\omega)$ through $x_{n m}^{(4)}(\omega)$ are unknown modal coefficients. Similarly, the general expression for normal surface velocity of each source can be written as

$$
\nu_{(\alpha)}\left(\theta_{\alpha}, \varphi_{\alpha}, \omega\right)=\sum_{n=0}^{\infty} \sum_{m=-n}^{n} V_{n m}^{(\alpha)}(\omega) P_{n}^{m}\left(\cos \theta_{\alpha}\right) \mathrm{e}^{i m \varphi_{\alpha}}(\alpha=1,2,3,4)
$$

where for a prescribed velocity distribution, Fourier coefficients $V_{n m}^{(1)}(\omega)$ through $V_{n m}^{(4)}(\omega)$ are known. 


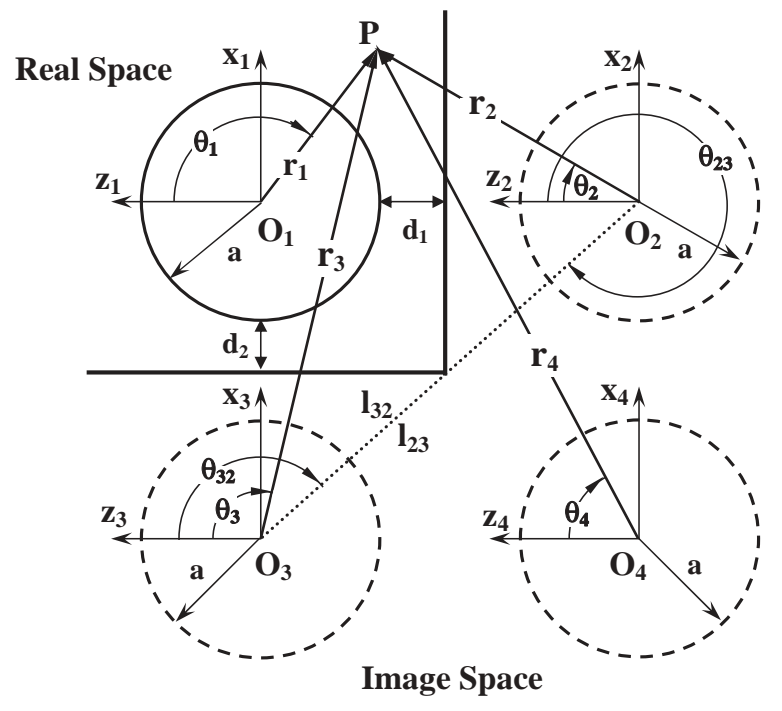

Fig. 2. Source-image configuration for the acoustic quarterspace problem.

$$
G_{n}\left(k r_{2}\right) P_{n}^{m}\left(\cos \theta_{2}\right) \mathrm{e}^{i m \varphi_{2}}=\sum_{p=0}^{\infty} \sum_{q=-p}^{p} Q_{p q n m} j_{p}\left(k r_{1}\right) P_{p}^{q}\left(\cos \theta_{1}\right) \mathrm{e}^{i q \varphi_{1}}
$$

where $G_{n}$ is one of the spherical Bessel functions of order $n$, and

$$
Q_{\mathrm{pqnm}}=\frac{2 i^{p-n}}{N_{p q}} \sum_{\sigma=|p-n|}^{p+n} i^{\sigma} b_{\sigma}^{n m p q} G_{\sigma}\left(k l_{12}\right) P_{\sigma}^{m-q}\left(\cos \theta_{12}\right) \mathrm{e}^{i(m-q) \varphi_{12}}
$$

Before imposing the appropriate boundary conditions, we first express the spherical wave functions of the $\left(r_{2}, \theta_{2}, \varphi_{2}\right)$ coordinate system in terms of spherical wave functions of the $\left(r_{1}, \theta_{1}, \varphi_{1}\right)$ coordinate system through application of the classical form of translational addition theorem for bi-spherical coordinates [7]:

where

$$
N_{p q}=\frac{2}{2 p+1} \cdot \frac{(p+q) !}{(p-q) !} b_{\sigma}^{n m p q}=(-1)^{q} \gamma_{\sigma}^{n m p q}(n, p, 0,0 \mid \sigma, 0)(n, p, m,-q \mid \sigma, m-q)
$$

in which $\gamma_{\sigma}^{n m p q}=\left[\frac{(n+m) !(p+q) !(\sigma-m+q) !}{(n-m) !(p-q) !(\sigma+m-q) !}\right]^{1 / 2}$ and the Clebsch-Gordan Coefficients are defined as [16]

$$
(n, p, m, q \mid \sigma, m+q)=\lambda_{\sigma}^{n m p q} \sum_{z}(-1)^{z} \xi_{\sigma z}^{n m p q}
$$

where

$$
\begin{aligned}
\xi_{\sigma z}^{n m p q}= & {[z !(n+p-\sigma-z) !(z+\sigma-n-q) !(p+q-z) !(z+\sigma-p+m) !(n-m-z) !]^{-1} } \\
\lambda_{\sigma}^{n m p a}= & {\left[\frac{2 \sigma+1}{(\sigma+n+p+1) !}(\sigma+n-p) !(n+p-\sigma) !(p+\sigma-n) ! \times(n-m) !(n+m) !(p-q) !(p+q)\right.} \\
& (\sigma+m+q) !(\sigma-m-q) !]^{1 / 2}
\end{aligned}
$$

where the summation must be performed over the non-negative values of $z$ that makes all factorials involving $z$ to be greater than or equal to zero. Furthermore, the associated Legendre function is defined so that $P_{n}^{-m}=$ $(-1)^{m}[(n-m) ! /(n+m) !] P_{n}^{m}(x)$.

Next, incorporation of the above addition theorem in Eq. (2) allows us to translate the wave components of each coordinate system in terms of spherical wave functions of the other coordinate systems, i.e., 


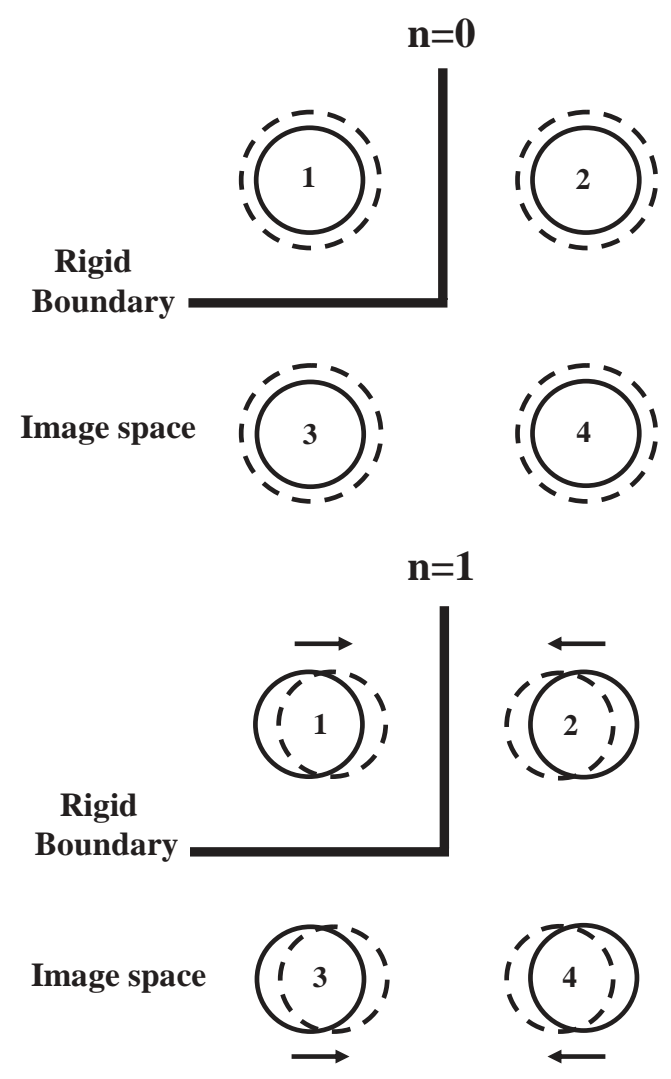

Fig. 3. Image of a pulsating and an oscillating source at a rigid corner.

$$
\Phi_{(\alpha)}\left(r_{\beta}, \theta_{\beta}, \varphi_{\beta}, \omega\right)=\sum_{n=0}^{\infty} \sum_{m=-n}^{n} X_{n m}^{(\alpha)}(\omega) h_{n}\left(k r_{\beta}\right) P_{n}^{m}\left(\cos \theta_{\beta}\right) \mathrm{e}^{i m \varphi_{\beta}} \quad(\alpha, \beta=1,2,3,4 ; \alpha \neq \beta)
$$

in which

$$
X_{n m}^{(\alpha)}(\omega)=\sum_{p=0}^{\infty} \sum_{q=-p}^{p} Q_{n m p q} x_{p q}^{(\alpha)}(\omega)
$$

where the unknown modal coefficients, $x_{p q}^{(\alpha)}(\omega)$, can be determined by imposing the pertinent boundary conditions, i.e., the continuity of radial velocity components at the surface of each source:

$$
\left.\left.w_{r}\left(r_{\alpha}, \theta_{\alpha}, \varphi_{\alpha}, \omega\right)\right]_{r_{\alpha}=a}=\frac{\partial \Phi\left(r_{\alpha}, \theta_{\alpha}, \varphi_{\alpha}, \omega\right)}{\partial r_{\alpha}}\right]_{r_{\alpha}=a}=\sum_{n=0}^{\infty} \sum_{m=-n}^{n} V_{n m}^{(\alpha)}(\omega) P_{n}^{m}\left(\cos \theta_{\alpha}\right) \mathrm{e}^{i m \varphi_{\alpha}}
$$

where $w_{r}\left(r_{\alpha}, \theta_{\alpha}, \varphi_{\alpha}, \omega\right)$ is the radial component of the fluid-particle-velocity vector and $\Phi\left(r_{\alpha}, \theta_{\alpha}, \varphi_{\alpha}, \omega\right)=$ $\sum_{\beta=1}^{4} \Phi_{(\beta)}\left(r_{\alpha}, \theta_{\alpha}, \varphi_{\alpha}, \omega\right)$. Substituting the velocity potential expansions Eqs (2) and (9) into the above boundary conditions yields

$$
V_{n m}^{(\alpha)}=-k h_{n}^{\prime}(k a) x_{n m}^{(\alpha)}(\omega)-k \sum_{\beta} j_{n}^{\prime}(k a) X_{n m}^{(\beta)}(\omega) \quad(\alpha, \beta=1,2,3,4 ; \alpha \neq \beta)
$$

The fluctuating acoustic pressure on the surface of the vibrating sphere constitutes its radiation loading. The radiation loading on the spherical surface excited in vibrational modes of various order (i.e., $n=0,1,2,3, \ldots$ ), as described 


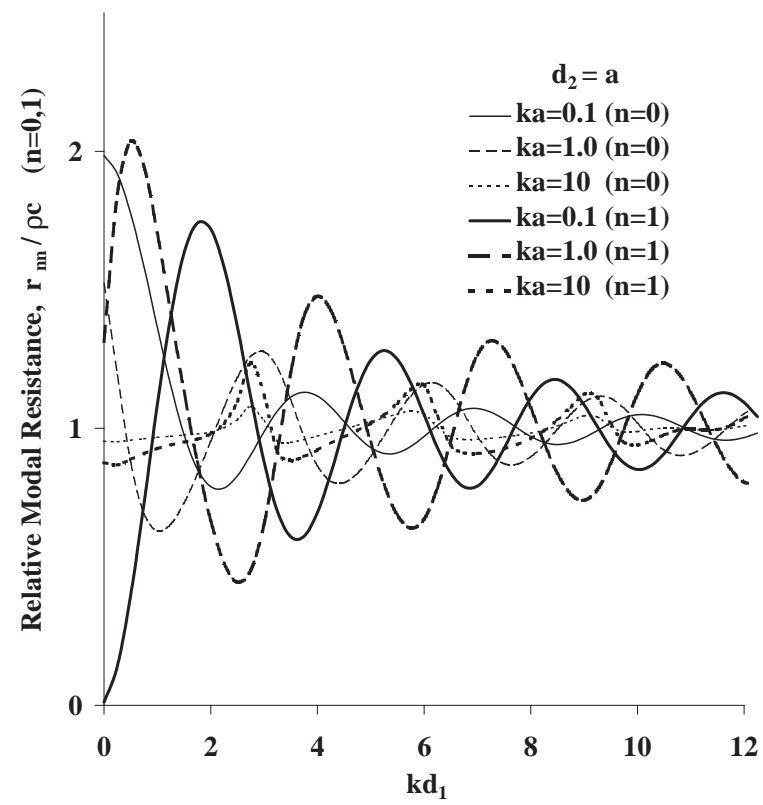

Fig. 4. Relative acoustic resistance for a pulsating and an oscillating source placed near the rigid boundary of an acoustic quarterspace at $d=a$ as a function of the separation parameter, $k d_{1}$, at selected excitation frequencies.

through an acoustic radiation impedance matrix, is determined in a few simple steps as follows. First, using second of Eqs (1), (2) and (9), acoustic pressure at the surface of each source is expressed as

$$
p_{(\alpha)}\left(r_{\alpha}=a, \theta_{\alpha}, \varphi_{\alpha}, \omega\right)=\sum_{n=0}^{\infty} \sum_{m=-n}^{n} P_{n m}^{(\alpha)}(\alpha, \omega) p_{n}^{m}\left(\cos \theta_{\alpha}\right) \mathrm{e}^{i m \varphi_{\alpha}}
$$

where

$$
p_{n m}^{(\alpha)}=-i \omega \rho h_{n}(k a) x_{n m}^{(\alpha)}(\omega)-i \omega \rho \sum_{\beta} j_{n}(k a) X_{n m}^{(\beta)}(\omega) \quad(\alpha, \beta=1,2,3,4 ; \alpha \neq \beta)
$$

which can readily be put in the matrix form

$$
\mathbf{P}=\mathbf{S c}
$$

where $\mathbf{S}$ is a $4(N+1)^{2} \times 4(N+1)^{2}$ square matrix that contains the coefficients multiplying the modal parameters $x_{n m}^{(\alpha)}$, and

$$
\begin{aligned}
\mathbf{p}= & {\left[p_{0,0}^{(1)}, p_{1,-1}^{(1)}, p_{1,0}^{(1)}, p_{1,1}^{(1)}, \ldots ; p_{0,0}^{(2)}, p_{1,-1}^{(2)}, p_{1,0}^{(2)}, p_{1,1}^{(2)}, \ldots ; p_{0,0}^{(3)}, p_{1,-1}^{(3)}, p_{1,0}^{(3)}, p_{1,1}^{(3)}, \ldots ;\right.} \\
& \left.p_{0,0}^{(4)}, p_{1,-1}^{(4)}, p_{1,0}^{(4)}, p_{1,1}^{(4)}, \ldots\right]^{T} \\
\mathbf{c}= & {\left[x_{0,0}^{(1)}, x_{1,-1}^{(1)}, x_{1,0}^{(1)}, x_{1,1}^{(1)}, \ldots ; x_{0,0}^{(2)}, x_{1,-1}^{(2)}, x_{1,0}^{(2)}, x_{1,1}^{(2)}, \ldots ; x_{0,0}^{(3)}, x_{1,-1}^{(3)}, x_{1,0}^{(3)}, x_{1,1}^{(3)}, \ldots ;\right.} \\
& \left.x_{0,0}^{(4)}, x_{1,-1}^{(4)}, x_{1,0}^{(4)}, x_{1,1}^{(4)}, \ldots\right]^{T}
\end{aligned}
$$

Next, the truncated system of Eq. (12) is expressed in the matrix form

$$
\mathbf{u}=\mathbf{R c}
$$

where $\mathbf{R}$ is a $4(N+1)^{2} \times 4(N+1)^{2}$ square matrix whose elements are the coefficients multiplying the modal parameters $x_{n m}^{(\alpha)}$ in the boundary conditions Eq. (12), and

$$
\begin{aligned}
\mathbf{u}= & {\left[V_{0,0}^{(1)}, V_{1,-1}^{(1)}, V_{1,0}^{(1)}, V_{1,1}^{(1)}, \ldots ; V_{0,0}^{(2)}, V_{1,-1}^{(2)}, V_{1,0}^{(2)}, V_{1,1}^{(2)}, \ldots ; V_{0,0}^{(3)}, V_{1,-1}^{(3)}, V_{1,0}^{(3)}, V_{1,1}^{(3)}, \ldots ;\right.} \\
& \left.V_{0,0}^{(4)}, V_{1,-1}^{(4)}, V_{1,0}^{(4)}, V_{1,1}^{(4)}, \ldots\right]^{T}
\end{aligned}
$$




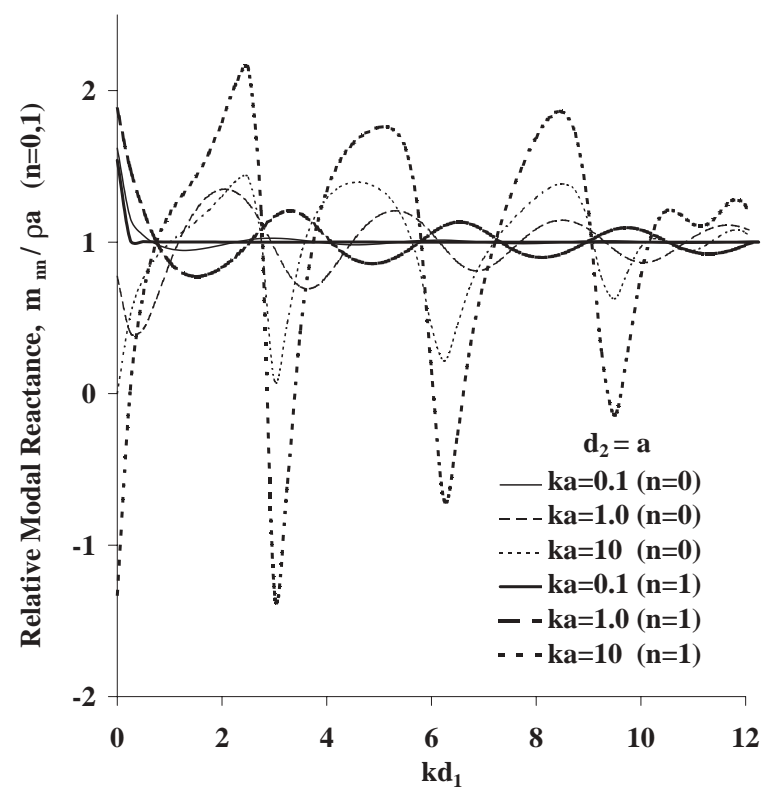

Fig. 5. Relative acoustic reactance for a pulsating and an oscillating source placed near the rigid boundary of an acoustic quarterspace at $d=a$ as a function of the separation parameter, $k d_{1}$, at selected excitation frequencies.

Incorporating Eq. (17) into Eq. (15), the modal surface pressures can simply be related to modal surface velocities by

$$
\mathbf{p}=\mathbf{Z u}
$$

where the modal acoustic radiation impedance matrix $Z$ is given as

$$
\boldsymbol{Z}=\boldsymbol{S} R^{-1}=\left[\begin{array}{llll}
\boldsymbol{Z}_{I, I} & \boldsymbol{Z}_{I, I I} & \boldsymbol{Z}_{I, I I I} & \boldsymbol{Z}_{I, I V} \\
\boldsymbol{Z}_{I I, I} & \boldsymbol{Z}_{I I, I I} & \boldsymbol{Z}_{I I, I I I} & \boldsymbol{Z}_{I I, I V} \\
\boldsymbol{Z}_{I I I, I} \boldsymbol{Z}_{I I I, I I} \boldsymbol{Z}_{I I I, I I I} \boldsymbol{Z}_{I I I, I V} \\
\boldsymbol{Z}_{I V, I} & \boldsymbol{Z}_{I V, I I} & \boldsymbol{Z}_{I V, I I I} & \boldsymbol{Z}_{I V, I V}
\end{array}\right]
$$

Noting the special structure of the impedance matrix, the $(N+1)^{2} \times(N+1)^{2}$ diagonal submatrices $\boldsymbol{Z}_{I, I}$ through $\boldsymbol{Z}_{I V, I V}$ essentially contain self-impedance elements corresponding to sound radiation from the first through the fourth source, respectively. The remaining off-diagonal submatrices take account of cross-impedance effects. Furthermore, each element of the above fully populated submatrices may beneficially be put in terms of a resistive and a reactive component as [17]:

$$
z_{n m}=(\omega)=r_{n m}(\omega)-i \omega m_{n m}(\omega)
$$

The most relevant acoustic radiation field quantities are pressure and intensity. Given the surface velocity distribution of each source (i.e., $V_{n m}^{(\alpha)}$ are known), one can determine the unknown modal coefficients using Eq. (17) (i.e., from $c=R^{-1} u$ ). Subsequently, the acoustic modal pressures may readily be obtained from Eq. (15), and the radial component of the acoustic power flux vector (acoustic intensity) radiated per unit solid angle from each sphere is found from [15]

$$
I_{\text {rad }}^{(\alpha)}=\frac{1}{2} \operatorname{Re}\left\{p\left(r_{\alpha}, \theta_{\alpha}, \varphi_{\alpha}, \omega\right) \times w_{r}^{*}\left(r_{\alpha}, \theta_{\alpha}, \varphi_{\alpha}, \omega\right)\right\}(\alpha=1,2,3,4)
$$

where the asterisk denotes a complex conjugate quantity, and 


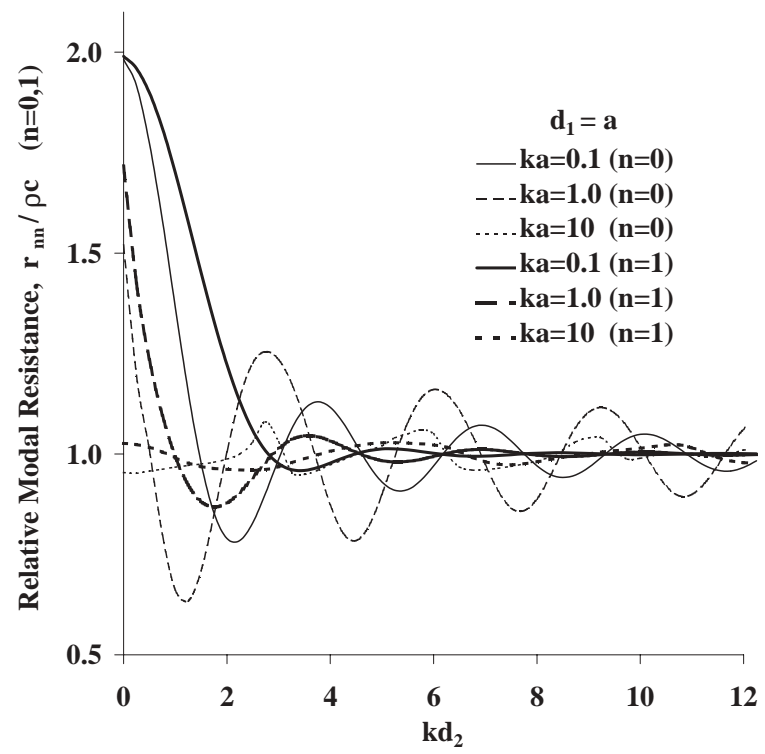

Fig. 6. Relative acoustic resistance for a pulsating and an oscillating source placed near the rigid boundary of an acoustic quarterspace at $d=a$ as a function of the separation parameter, $k d_{2}$, at selected excitation frequencies.

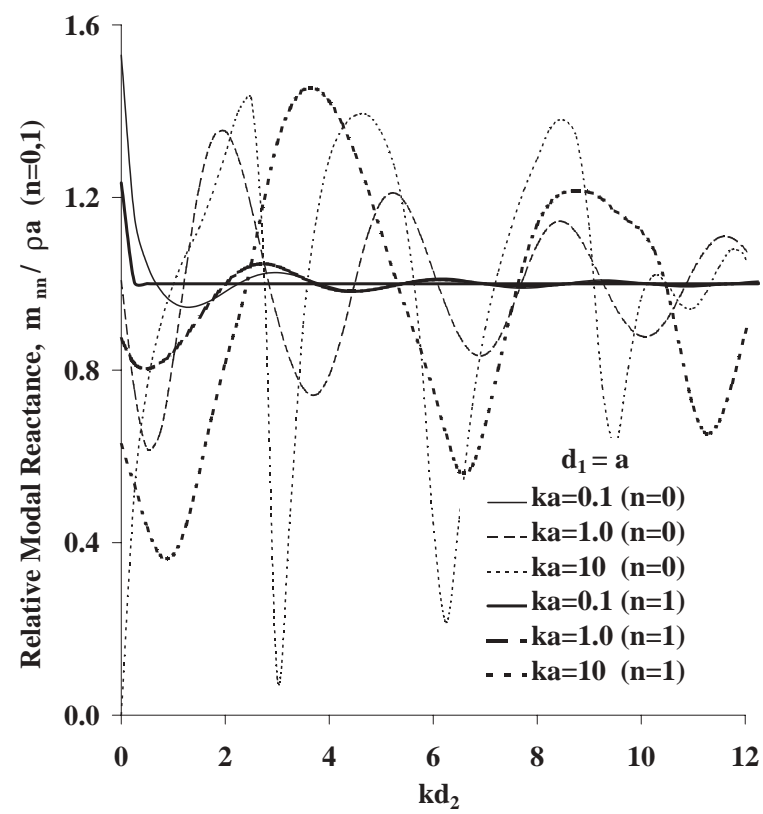

Fig. 7. Relative acoustic reactance for a pulsating and an oscillating source placed near the rigid boundary of an acoustic quarterspace at $\mathbb{q}=a$ as a function of the separation parameter, $k d_{2}$, at selected excitation frequencies.

$$
\begin{aligned}
p\left(r_{\alpha}, \theta_{\alpha}, \varphi_{\alpha}, \omega\right) & =-i \omega \rho \sum_{\beta=1}^{4} \Phi_{(\beta)}\left(r_{\alpha}, \theta_{\alpha}, \varphi_{\alpha}, \omega\right) \\
w_{r}\left(r_{\alpha}, \theta_{\alpha}, \varphi_{\alpha}, \omega\right) & =-\frac{\partial}{\partial r_{\alpha}} \sum_{\beta=1}^{4} \Phi_{(\beta)}\left(r_{\alpha}, \theta_{\alpha}, \varphi_{\alpha}, \omega\right)
\end{aligned}
$$



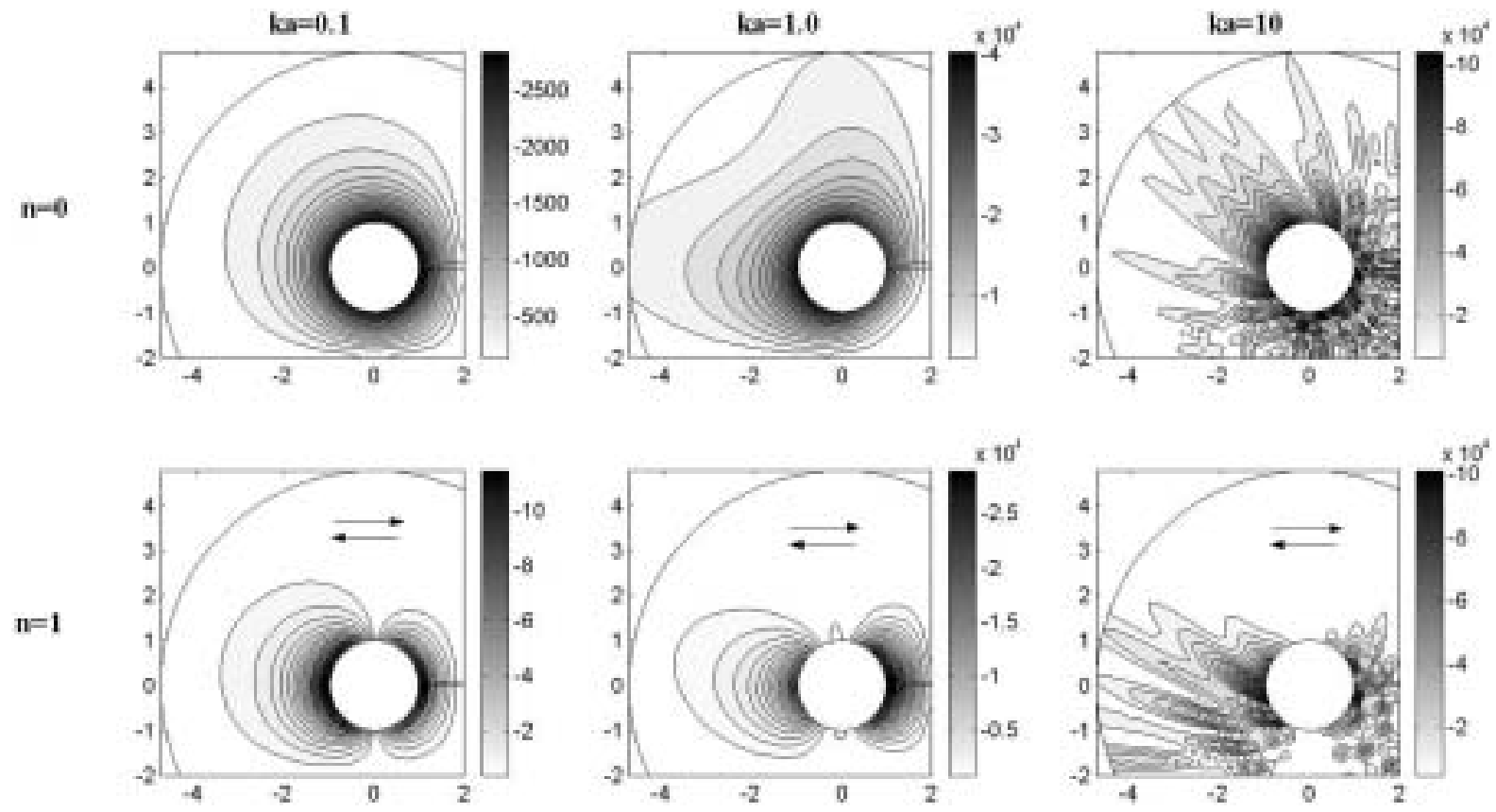

Fig. 8. Radiation intensity distribution in the vicinity of the spherical source located one radius away from the quarterspace boundary $\left(d_{1}=d_{2}=a\right)$ at selected excitation frequencies.

\section{Numerical results and discussion}

In order to illustrate the nature and general behaviour of the solution, we consider a numerical example in this section. The surrounding ambient fluid is assumed to be water at atmospheric pressure and 300 kelvin $(\rho=$ $\left.0.997 \mathrm{~g} / \mathrm{cm}^{3}, c=149700 \mathrm{~cm} / \mathrm{s}\right)$. Each source is assumed to be of dimension $\alpha=1 \mathrm{~cm}$, which may vibrate in the pulsating $(n=0)$ or the translational oscillating $(n=1)$ mode. his choice is sensible because the pulsating and the translational oscillating radiators are well-known to best represent the practical "expander" and "shaker" type acoustic transducers, respectively [1,8-14]. Accurate computation of Bessel functions is achieved by employing MATLAB specialized math functions "besselh" and "besselj." The precision of calculated values was checked against the printed tabulations in the handbook by Abramowitz and Stegun [16]. Subsequently, a MATLAB code was constructed for treating the boundary value problem, to determine the unknown modal coefficients, and to compute the modal im[edance matrix, $Z=S R^{-1}$, and other relevant acoustic quantities as functions of the distance parameters $k d_{1}, k d_{2}$ and the nondimensional excitation frequency $k a=\omega a / c$. Accurate computations for derivatives of spherical Bessel functions were accomplished by utilizing (10.1.19) and (10.1.22) in [16]. The computations were performed on a Pentium IV personal computer with a truncation constant of $N=7$ to assure convergence in the high frequency range, and also in case of close proximity of the source to the quarterspace boundary. Thus the largest (complex) matrices used were $256 \times 256$. Furthermore, as illustrated in Fig. 3, in order to satisfy the rigid boundary conditions (i.e., elimination of the normal component of the fluid velocity on the fictitious boundary), we must incorporate $V_{00}^{(1)}=V_{00}^{(2)}=V_{00}^{(3)}=V_{00}^{(4)}$ for the pulsating $(n=0)$ source in the above computations. Likewise, for the translational oscillating $(n=1)$ source we have to take the condition $V_{10}^{(1)}=-V_{10}^{(2)}=V_{10}^{(3)}=-V_{10}^{(4)}$ into account $[14,18]$.

Figures 4 through 7 show the effects of the distance parameters, $k d_{1}$ (with $d_{2}=a$ ), and $k d_{2}$ (with $d_{1}=a$ ), on the resistive and the reactive components of acoustic radiation load on the pulsating and the translational oscillating radiator at selected excitation frequencies of $k a=0.1,1$, and 10 . Here we note that the resistive and the reactive components of the modal impedances are each normalized to their corresponding values in an infinite unbounded medium, i.e., to the value when the source is infinitely far removed from the corner (i.e., $d_{1}=d_{2}=100 a$ ). Examination of these figures leads to the following observations. First, for the pulsating source at small $k a$ (i.e., 

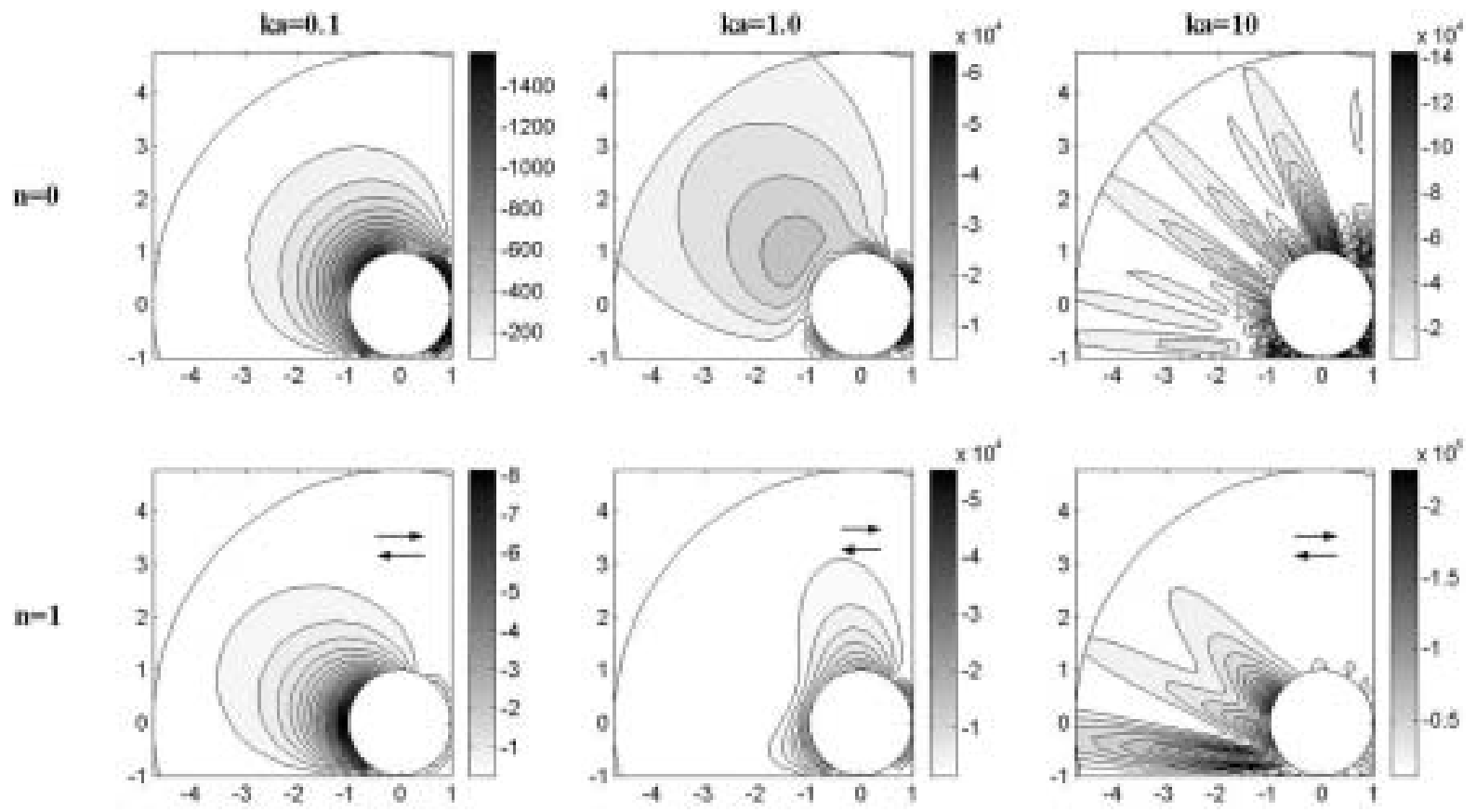

Fig. 9. Radiation intensity distribution in the vicinity of the spherical source touching the quarterspace boundary $\left(d=d_{2}=0\right.$ at selected excitation frequencies.

point source limit), the resistance, and therefore the power output, tends to effectively double as the separation parameters $k d_{1}$ and $k d_{2}$ are minimized [19]. Similarly, for translational oscillations at small $k a$, the modal resistance values tend to zero (double) as the separation parameter $k d_{1}\left(k d_{2}\right)$ is minimized. Furthermore, the modal impedance components in the $d_{2}=a$ case Figs 4 and 5 show vely more oscillations with respect to the distance parameter in comparison to $d_{1}=a$ situation. Lastly, at the highest frequency considered (i.e., $k a=10$ ) for $d_{2}=a$ Fig. 5 , a negative modal reactance is observed for some relatively narrow ranges of the distance parameter which implies that the reactance becomes stiffness controlled in these regions [17].

To further assess the effect of presence of the rigid corner on the radiated acoustic field, the radiation intensity distribution $\left(\mathrm{W} / \mathrm{cm}^{2}\right)$ at selected nondimensional frequencies in the neighbourhood of the spherical source that is one radius away from (almost touching) the boundary are presented in Fig. 8 (Fig. 9). It is very interesting to study the change in strength and directionality of the radiated energy as the source frequency, position, and mode of oscillations are changed. At $k a=0.1$ for $d_{1}=d_{2}=a$ for Fig. 8, proximity of the source to the rigid corner does not seem to be very influential and the relatively small amplitude radiated sound energy is almost evenly distributed around the source. Furthermore, the radiation intensity magnitudes for the pulsating $(n=0)$ source are significantly higher than the corresponding values for the translational oscillating $(n=1)$ radiator. At the intermediate frequency of $k a=$ 1 , the existence of the rigid corner begins to show its effect as the directio of the radiated sound energy somewhat enhances while its overall magnitude drastically increases, especially for the translational oscillating radiator ( $n=$ 1). At the highest frequency $(k a=10)$, the energy reflected from the rigid corner dominates the acoustic field, as there is a radical increase in the directionality of the radiated energy. Furthermore, at this frequency sound energy is mainly distributed around the bisecting $\left(d_{1}=d_{2}\right)$ line in the $n=0$ case. This is in contrast to the translational oscillating $(n=1)$ situation in which backward/forward sound radiation (i.e., parallel to the horizontal boundary) prevails. When the source is almost touching the corner Fig. 9 the acoustic interactions with the rigid corner naturally become more dominant at all frequencies and remarks as in above discussion can be made.

Finally, to verify overall validity of the work we consider the problem of a source in an acoustic halfspace by making separate computations for the limiting cases of $d_{1}=100 a$ and $d_{2}=100 a$. The relative modal impedance components versus the separation parameter $k r_{0}\left(r_{0}=d_{2}\right.$ or $\left.d_{1}\right)$ at selected nondimensional frequencies 


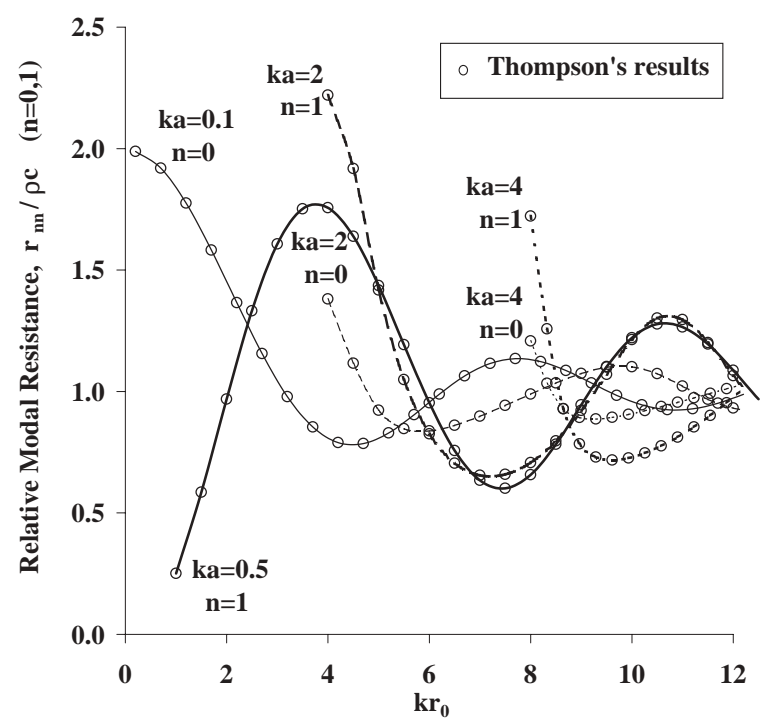

Fig. 10. Relative modal resistance components versus the separation parameter in the acoustic halfspace limit $\left(k 0=k d_{1} o r k d_{2}\right.$, and $d_{2}=$ $100 a$ or $d_{1}=100 a$, respectively).

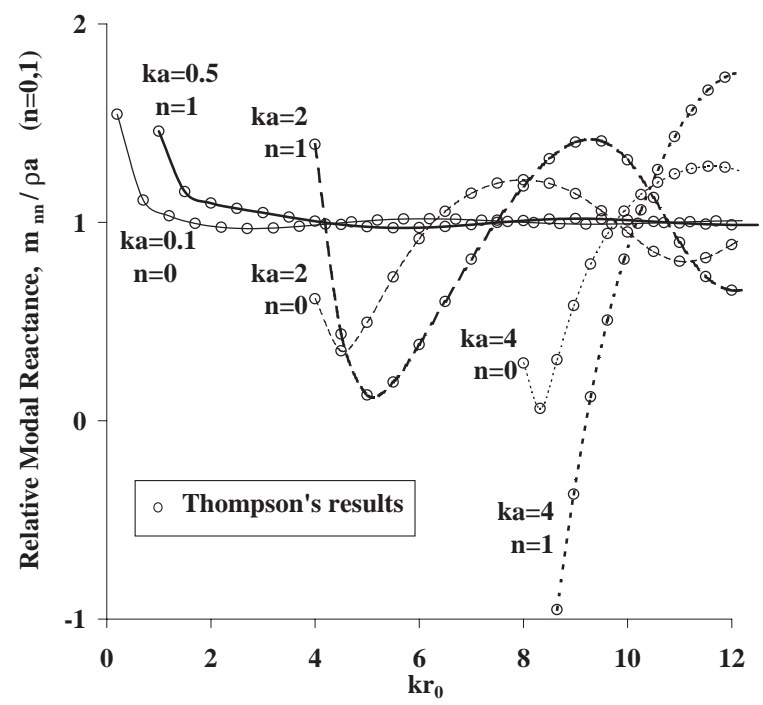

Fig. 11. Relative modal reactance components versus the separation parameter in the acoustic halfspace limit $\left(k 0=k d_{1} o r k d_{2}\right.$, and $d_{2}=$ $100 a$ or $d_{1}=100 a$, respectively).

are calculated. Figures 10 and 11 show that excellent agreements are obtained with the numerical results presented in [9]. Further verifications were made with the numerical results offered in [18].

\section{Conclusions}

Acoustic radiation impedance curves have been generated for modal vibrations of a spherical source immersed near the rigid boundary of an acoustic quarterspace. These curves are product of an exact multi-scattering treatment that involves utilization of the translational addition theorem for spherical wave functions in combination with the classical method of images. Numerical results reveal the important effects of source position, excitation frequency, 
and surface velocity distribution on the acoustic radiation impedance load and the radiation intensity distribution. The presented formulation can lead to a better understanding of dynamic response of near-corner sources that are of practical interest in room acoustics, outdoor acoustics, and noise control engineering.

\section{Acknowledgements}

The authors wish to sincerely thank professor John A. Roumeliotis for valuable consultations on theory of translational addition theorems for spherical wave functions.

\section{References}

[1] R. New and T.J. Eisler, Acoustic Radiation from Multiple Spheres, J. Sound Vib 22 (1972), 1-17.

[2] G.C. Gaunaurd, H. Huang and H.C. Strifors, Acoustic Scattering by a Pair of Spheres, J. Acoust. Soc. Am. 98 (1995), $495-507$.

[3] P. Gabrelli and M. Mercier-Findori, Acoustic Scattering by Two Spheres: Multiple Scattering and Symmetry Considerations, J. Sound Vib. 241 (2001), 423-439.

[4] M.I. Karnovskii, Interaction Acoustical Impedance of Spherical Radiators and Resonators, C. R. (Dokl.) Acad. Sci. URSS 32 (1941), 40-43.

[5] M.I. Karnovskii, Calculation of the Radiation Resistance of Several Types of Distributed Radiator Systems, Soviet Physics-Acoustics 2 (1956), 280-293

[6] U. Ingard and Jr. G.L. Lamb, Effect of a Reflecting Plane on the Output of Sound Sources, J. Acoust. Soc. Am. 29 (1957), $743-744$.

[7] Y.A. Ivanov, Diffraction of Electromagnetic Waves on Two Bodies, National Aeronautics and Space Administration, Washington, D.C., 1970 .

[8] W. Thompson, Jr., Radiation from a Spherical Acoustic Source Near a Scattering Sphere, J. Acoust. Soc. Am. 60 (1976), $781-787$.

[9] W. Thompson, Jr., Acoustic Coupling Between two Finite-sized Spherical Sources, J. Acoust. Soc. Am. 62 (1977), 8-11.

[10] W. Thompson, Jr., Acoustic Coupling Between a Pulsating and an Oscillating Sphere, J. Acoust. Soc. Am. 74 (1983), 1148-1050.

[11] W. Thompson, Jr., Acoustic Radiation from a Spherical Source Embedded Eccentrically within a Fluid Sphere, J. Acoust. Soc. Am. 54 (1973), 1694-1707.

[12] S.A. Lease and W. Thompson, Jr., Use of Translational Addition Theorems for Spherical Wave Functions in Nonaxisymmetric Acoustic Field Problems, J. Acoust. Soc. Am. 90 (1991), 1155-1160.

[13] S.A. Lease and W. Thompson, Jr., Nonaxisymmetric Acoustic Radiation from a Pair of Sources Embedded in a Fluid Sphere, J. Acoust. Soc. Am. 90 (1991), 1161-1166.

[14] S.M. Hasheminejad, Modal Acoustic Force on a Spherical Radiator in an Acoustic Halfspace with Locally Reacting Boun dary, Acta acustica-Acustica 87 (2001), 443-453.

[15] A.D. Pierce, Acoustics; An Introduction to Its Physical Principles and Applications, American Institute of Physics, New York, 1991.

[16] M. Abramowitz and I.A. Stegun, Handbook of Mathematical Functions, National Bureau of Standards, Washington, DC, 1965.

[17] M.C. Junger and D. Feit, Sound, Structures, and Their Interaction, 2nd ed., MIT Press, Massachusetts, 1986.

[18] S.M. Hasheminejad and T.L. Geers, Doubly Asymptotic Approximations for an Acoustic Halfspace, ASME J. Vibration and Acoustics 114 (1992), 555-563.

[19] E. Skudrzyk, The Foundations of Acoustics, Springer-Verlag, New York, 1971. 

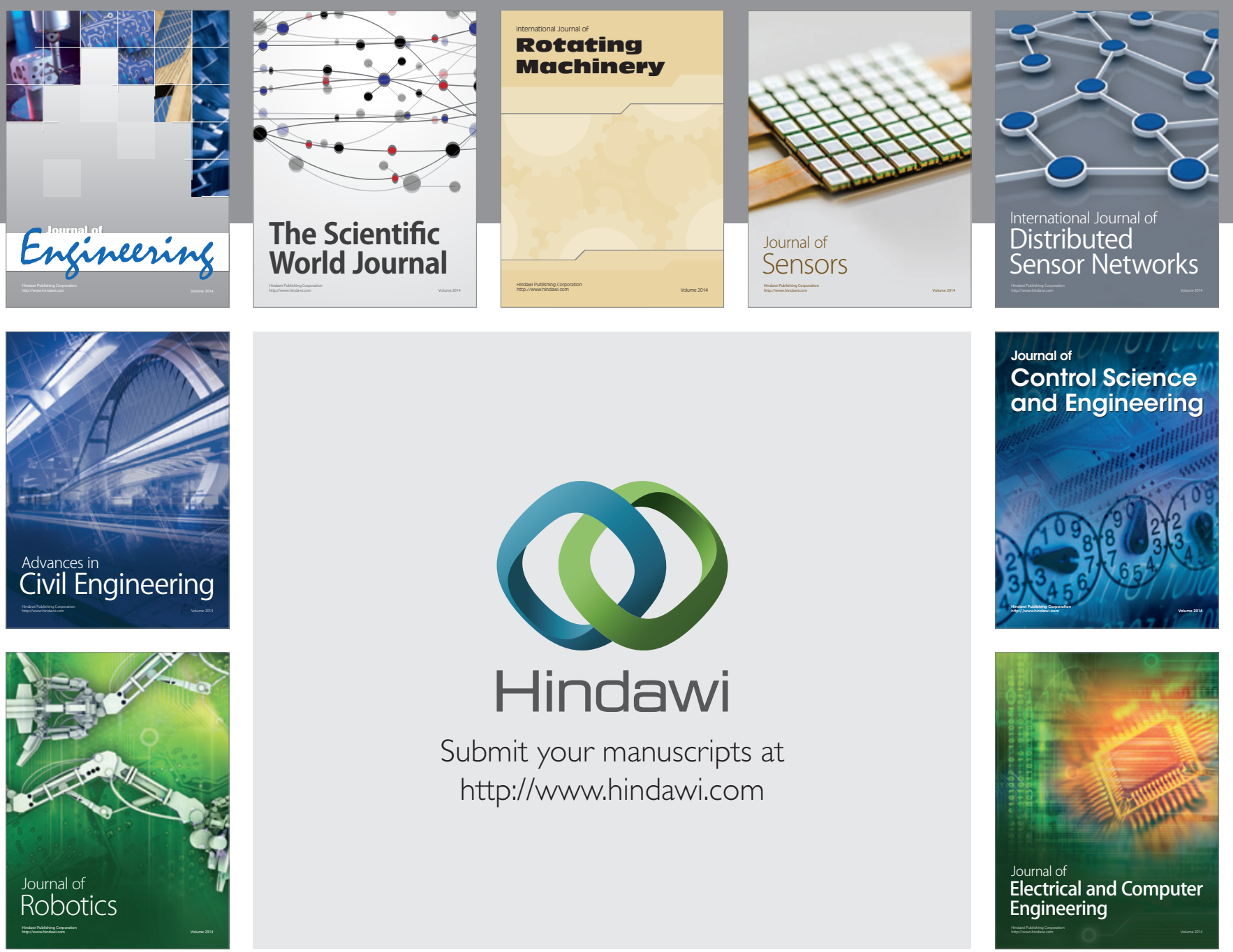

Submit your manuscripts at

http://www.hindawi.com
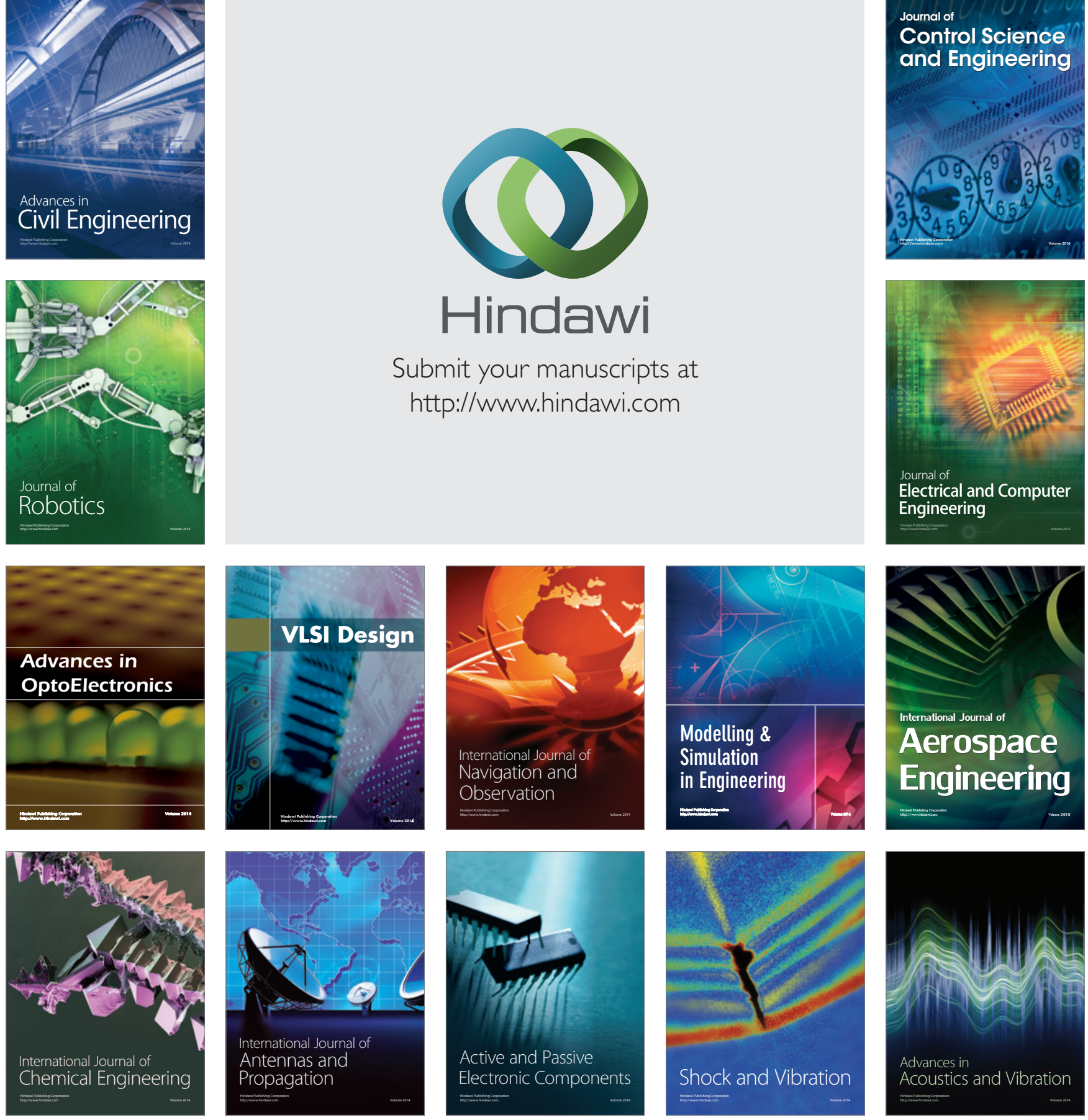